\title{
Survey of glucosinolate variation in leaves of Brassica rapa crops
}

\author{
Bo Yang · Carlos F. Quiros
}

Received: 15 October 2009/Accepted: 10 March 2010/Published online: 31 March 2010

(C) The Author(s) 2010. This article is published with open access at Springerlink.com

\begin{abstract}
The breakdown products of glucosinolates (gsl) are biologically active secondary metabolites involved in plant defense and human nutrition. We identified and quantified 14 different gsl present in the young leaves of 82 different varieties of Brassica rapa, including the following crops: Chinese cabbage, broccoleto, Pak choi and other leafy vegetables, turnip, sarson and rapeseed. We did not find crop specific gsl, but their quantity varied extensively among varieties and crops, except that the Chinese cabbage accessions tended to have similar gsl profile and amount. Gluconapin, glucobrassicanapin (aliphatic), neoglucobrassicin, glucobrassicin (indolic), and gluconaturtiin (aromatic) are the predominant gsl in most of the varieties surveyed. We also found two gsl not commonly found in B. rapa, 2-methyl-2-propenyl and $n$-butyl. Their identities were confirmed by HPLCMS. Most of the Chinese cabbages contain lower amount of aliphatic than indolic gsl, whereas broccoleto, turnip and rapeseed all have much higher aliphatic gsl content than indolic gsl content. The predominant aliphatic gsl in most of the varieties contain 4-carbon side-chains. The lack of significant correlation
\end{abstract}

Electronic supplementary material The online version of this article (doi:10.1007/s10722-010-9549-5) contains supplementary material, which is available to authorized users.

B. Yang · C. F. Quiros ( $)$

Department of Plant Sciences, University of California, Davis, CA 95616, USA

e-mail: cfquiros@ucdavis.edu observed between the conversion of 3- to 4-carbon side-chain gsl and the conversion of 4- to 5-carbon side-chain gsl suggests that these two elongation cycles are probably under the control of two independent genes in B. rapa. The absence of glucoraphanin in all accessions indicates that only functional $\mathrm{Brgsl}$-Alk alleles are present in B. rapa.

Keywords Brassica rapa - Brassicaceae . Glucosinolates $\cdot$ Secondary metabolites

\section{Introduction}

Glucosinolates (gsl) have been of considerable interest to plant biologists for many years. These nitrogen- and sulfur-containing plant secondary metabolites and their breakdown products are responsible for the distinctive flavor and taste of the plants and crops that contain them. Certain types of gsl derivatives, such as the isothiocyanate sulforaphane, have been found to possess biological activity as cancer-prevention agents in mammals, and others as plant defense compounds against insect and diseases (Mithen 2001).

In Arabidopsis thaliana (L.) Heynh., a Brassica relative, it was reported that gsl accumulation varies significantly among different organs and developmental stages, with regards to both composition and concentration. The highest gsl concentrations were found in young leaves and reproductive organs such 
as seeds, siliques, flowers and developing inflorescences (Brown et al. 2003). Exceptionally high concentration of gsl was reported in the sulfur-rich S-cells of the flower stalk (Koroleva et al. 2000). This was the first report of gsl accumulation localized to a specific cell type found just outside the phloem. Roots, stems and fully expanded leaves contain intermediate concentrations of gsl. The lowest concentrations were found in senescing rosette leaves (Brown et al. 2003, Petersen et al. 2002).

With the completion of the A. thaliana genome sequence, gsl research progressed rapidly in the past decade (Halkier and Gershenzon 2006). The availability of abundant functional genomic resources along with the large natural gsl variation among ecotypes in A. thaliana has greatly facilitated this research. Much of the core biosynthetic pathway of these compounds, their breakdown pathway and their regulation factors in A. thaliana has been elucidated. However, this knowledge is just starting to being transferred to various gsl-containing vegetable crops included in human diet. A few major genes involved in the aliphatic, methionine-derived gsl biosynthesis in B. oleracea have been genetically characterized and cloned (Li and Quiros 2002; Li and Quiros 2003; Gao et al. 2004). Gsl content have been surveyed in several crops such as broccoli, broccoli sprouts, cauliflower and oilseed rape among others (Barillari et al. 2005; Windsor et al. 2005; Font et al. 2005; Tian et al. 2005; Skutlarek et al. 2004; Branca et al. 2002).

The species B. rapa L. em. Metzg. includes a great array of crops commonly used in human diet, particularly in Asian cuisine, such as Chinese cabbage, pak-choi, among many other leafy vegetables, as well as turnip and rapeseed. Limited evaluations of gsl have been performed in different tissues of a few B. rapa crops with the objective of determining their potential biological activity (Siemens et al. 2002; Cipollini et al. 2003; Kang et al. 2006; Bellosta et al. 2007). Padilla et al. (2007) determined the gsl diversity of $B$. rapa varieties restricted to turnip greens from northwestern Spain. In the present study, we analyzed gsl profiles from young leaves of 82 different $B$. rapa accessions representing all major crops and constituting a diverse sample of the genetic, geographical and environmental range of this species. We found extensive variation in both the composition and total concentration of gsl in the accessions of this species.

\section{Materials and methods}

Plant material

Eighty two varieties (Table 1), representing most major crops of $B$. rapa, were sampled in the present study. All accessions come from the Brassica germplasm working collection at the Department of Plant Sciences, UC Davis. These include the following subspecies: B. rapa ssp. pekinensis (Lour.) Hanelt (Chinese cabbage), B. rapa ssp. chinensis (L.) Hanelt (pak choi), B. rapa ssp. nipposinica (L. H. Bailey) Hanelt (syn. B. ruvo Bailey) (broccoleto), B. rapa ssp. rapifera Metzg. (turnip), B. rapa ssp. perviridis L. H. Bailey (Japanese/Oriental tender green), $B$. rapa ssp. oleifera (DC.) Metzg. (rapeseed), B. rapa ssp. dichotoma (Roxb.) Hanelt (toria) and B. rapa ssp. trilocularis (Roxb.) Hanelt (Yellow sarson). Seeds from each accession were germinated in $12 \times 20 \times 1.5$ inch trays (135 wells in each tray) and transferred into $6.5 \times 6.5 \times 7$ inch pots at 3 weeks. The trays and pots were filled with "Super Soil” potting mix (Rod McLellan Co., San Mateo, $\mathrm{CA}$ ) and the plants were grown in the greenhouse. The natural photoperiod was extended to $16 \mathrm{~h}$ by metal halide light bulbs (Agrosun $400 \mathrm{~W}$, Philips). Temperature was maintained at $24^{\circ} \mathrm{C}$ day; $18^{\circ} \mathrm{C}$ night. The leaf tissue was harvested from each plant 6 weeks after germination. $200 \mathrm{mg}$ of leaf material per sample was used for the gsl extraction. All samples for each variety were analyzed in triplicate in independent experiments.

\section{Glucosinolate extraction and purification}

The purification technique used was modified from the basic Sephadex/sulfatase Arabidopsis protocol previously described by Kliebenstein et al. (2001a, b). Each $200 \mathrm{mg}$ fresh leaf tissue sample was frozen in liquid nitrogen and ground into fine powder in a $1.5 \mu$ l eppendorf tube with a mini pestle. $500 \mu \mathrm{l}$ of $90 \%$ methanol was added to the fine powder, followed by incubation at $80^{\circ} \mathrm{C}$ for $10 \mathrm{~min}$. After incubating at room temperature for $1 \mathrm{~h}$, the tissue and protein were precipitated by centrifugation for $10 \mathrm{~min}$ at $3,500 \mathrm{rpm}$ and the supernatant used for anion-exchange chromatography. Each well of a 96 well filter plate (ISC BioExpress) was loaded with Sephadex DEAE A-25 using the multiscreen filter 
Table 1 List of Brassica rapa accession sampled in this study

\begin{tabular}{|c|c|c|}
\hline Acc & Crop variety & Origin \\
\hline 1 & Rapid cycling 121 & Wisconsin, USA \\
\hline 2 & Chinese cabbage b, Beijing Fanxinbai & Beijing, China \\
\hline 3 & Chinese cabbage, Lubao F1 Hybrid & Beijing, China \\
\hline 4 & Chinese cabbage, Zhongfeng F1 Hybrid & Beijing, China \\
\hline 5 & Chinese cabbage, 1063 , land race & Beijing, China \\
\hline 6 & Chinese cabbage, 282 Michihli F-3X & California, USA \\
\hline 7 & Chinese cabbage, 50 & Beijing, China \\
\hline 8 & Chinese cabbage, 536, land race & Beijing, China \\
\hline 9 & Chinese cabbage, 582, land race & Beijing, China \\
\hline 10 & Chinese cabbage, 76 & Beijing, China \\
\hline 11 & Chinese cabbage, Bianzao-26 & Henan, China \\
\hline 12 & Chinese cabbage, CC20-1 & Henan, China \\
\hline 13 & Chinese cabbage, CC20-2 & Henan, China \\
\hline 14 & Chinese cabbage, CC20-3 & Henan, China \\
\hline 15 & Chinese cabbage, CC20-4 & Henan, China \\
\hline 16 & Chinese cabbage, CC $35-1$ & Henan, China \\
\hline 17 & Chinese cabbage, CC35-2 & Henan, China \\
\hline 18 & Chinese cabbage, CC $35-4$ & Henan, China \\
\hline 19 & Chinese cabbage, CC $35-5$ & Henan, China \\
\hline 20 & Chinese cabbage, CC $35-6$ & Henan, China \\
\hline 21 & Chinese cabbage, China Pride \#33 F1 & Japan \\
\hline 22 & Chinese cabbage, Early Jade Pagoda F1 & Japan \\
\hline 23 & Chinese cabbage, Early Top F1 & Japan \\
\hline 24 & Chinese cabbage, F1 \#32 & Japan \\
\hline 25 & Chinese cabbage, Guang 90 E 16 & Henan, China \\
\hline 26 & Chinese cabbage, Hsia Shang (35 days) & Taiwan \\
\hline 27 & Chinese cabbage, Kwan-Hoo Choi & Korea \\
\hline 28 & Chinese cabbage, Market Pride & Japan \\
\hline 29 & Chinese cabbage, Matsushima & Maine, USA \\
\hline 30 & Chinese cabbage, Michihili Jade Pagoda F1 & Japan \\
\hline 31 & Ch. cabbage landrace & Taiwan \\
\hline 32 & Chinese cabbage, Oriental King F1 & Japan \\
\hline 33 & Chinese cabbage, Ri29-3 & Henan, China \\
\hline 34 & Chinese cabbage, Spring Triumph \#27 F1 & Japan \\
\hline 35 & Chinese cabbage, Tip top F1 & Japan \\
\hline 36 & Chinese cabbage, Tropical Delight F1 & Japan \\
\hline 37 & Chinese cabbage, Tropical Pride (F1) & Japan \\
\hline 38 & Chinese cabbage, Winter Giant F1 & Japan \\
\hline 39 & Chinese cabbage, Winter Knight \#17 & Japan \\
\hline 40 & Chinese cabbage, Wong Bok & Japan \\
\hline 41 & Broccoletto, Sessantina A Cima Grande & Wellesbourne, UK \\
\hline 42 & Broccoletto, Cima Di Rapa & Wellesbourne, UK \\
\hline 43 & Japanese green, Shirona & Wellesbourne, UK \\
\hline 44 & Oriental green, Koyona (Mizuna) & Maine, USA \\
\hline 45 & Pak Choi, Lei-Choi & California, USA \\
\hline
\end{tabular}


Table 1 continued

\begin{tabular}{|c|c|c|}
\hline Acc & Crop variety & Origin \\
\hline 46 & Pak Choi, Japanese Greens, Taisai & Wellesbourne, UK \\
\hline 47 & Pak Choi, Chinese Cabbage & Wellesbourne, UK \\
\hline 48 & Turnip Destro & Japan \\
\hline 49 & Turnip Kamo Kabu & Japan \\
\hline 50 & Turnip, Kanamachi Kokabu & Japan \\
\hline 51 & Turnip, Kenshin Kabu & Japan \\
\hline 52 & Turnip, Leaf Mustard & Japan \\
\hline 53 & Turnip, Market Express (F1) & Japan \\
\hline 54 & Turnip unnamed & Wellesbourne, UK \\
\hline 55 & Turnip, Navet Rave De Treignac & Wellesbourne, UK \\
\hline 56 & Turnip, Ndzawana & Japan \\
\hline 57 & Turnip, Togari Kabu & Japan \\
\hline 58 & Turnip, Zaruishi & Japan \\
\hline 59 & Turnip Rapa Palla di Neve & Wellesbourne, UK \\
\hline 60 & Turnip Royal Crown \#1 (F1) & Japan \\
\hline 61 & Turnip Royal Crown \#2 (F1) & Japan \\
\hline 62 & Turnip Tokyo Market Second Early & Japan \\
\hline 63 & Turnip Tokyo Top (F1) & Japan \\
\hline 64 & Turnip White Knight (F1) & Japan \\
\hline 65 & Turnip Yorii Spring & Japan \\
\hline 66 & Wild rapeseed 770010 & Japan \\
\hline 67 & Wild rapeseed 770016 & Japan \\
\hline 68 & Wild rapeseed 770024 & Japan \\
\hline 69 & Wild rapeseed 770072 & Japan \\
\hline 70 & Wild rapeseed 771003 & Turkey \\
\hline 71 & Wild rapeseed 771042 & Sweden \\
\hline 72 & Wild rapeseed 771048 & Afghanistan \\
\hline 73 & Wild rapeseed 771059 & Iran \\
\hline 74 & Wild rapeseed 771072 & Thailand \\
\hline 75 & Wild rapeseed 771073 & USSR \\
\hline 76 & Wild rapeseed 771091 & Montana, USA \\
\hline 77 & Wild rapeseed 770140 & Pakistan \\
\hline 78 & Wild rapeseed 771006 & India \\
\hline 79 & Wild rapeseed 771148 & USSR \\
\hline 80 & Toria $770278 \mathrm{~K} 940$ & Pakistan \\
\hline 81 & Yellow Sarson 770407K899 & Pakistan \\
\hline 82 & Yellow Sarson R2L & Wisconsin, USA \\
\hline
\end{tabular}

plate column loader (Millipore). $300 \mu \mathrm{l}$ of water was added to each well and allowed to equilibrate for $1 \mathrm{~h}$. The filter plate was placed on top of a deep well 96 well plate. After water was spun out to the plate by centrifugation for $2 \mathrm{~min}$ at $1,000 \mathrm{rpm}, 200 \mu \mathrm{l}$ of the supernatant was added to the 96-well Sephadex columns and the liquid spun into the plate by centrifuging for $3 \mathrm{~min}$ at $1,200 \mathrm{rpm}$ at room temperature. All the liquid in the plate was discarded. Then the Sephadex columns were washed one time with $175 \mu \mathrm{l}$ of $90 \%$ methanol, and two times with $150 \mu \mathrm{l}$ of water. To desulfate gsl in the column, $10 \mu \mathrm{l}$ of sulfatase solution and $70 \mu \mathrm{l}$ of water were added to each column and the plate placed in the dark for overnight sulfatase incubation at room temperature. Desulfo-gsl were eluted by centrifuging for $3 \mathrm{~min}$ at 
$1,200 \mathrm{rpm}$ at room temperature placing a shallowwell $2 \mathrm{ml}$ 96-well plate (Agilent Technologies) in the bottom of the filter column plate.

\section{HPLC analysis}

Twenty microliters of the gsl extract was run on a 5- $\mu \mathrm{m}$ column (Lichrospher 100 RP-18 end capped, $250 \mathrm{~mm} \times 4.6 \mathrm{~mm}$, Alltech) on an Agilent 1100 series High Performance Liquid Chromatography (HPLC). Compounds were detected by UV at $229 \mathrm{~nm}$ and separated utilizing the following program with a water-acetonitrile gradient at a constant $1.00 \mathrm{ml} \mathrm{m^{-1 }}$ flow rate. The program included a 5-min gradient from 1.5 to $7.0 \%(\mathrm{v} / \mathrm{v})$ acetonitrile, a 5 min gradient from 7.0 to $25.0 \%(\mathrm{v} / \mathrm{v})$ acetonitrile, a 4 min gradient from 25.0 to $80.0 \%$ (v/v) acetonitrile, $3 \mathrm{~min}$ at $80.0 \%(\mathrm{v} / \mathrm{v})$ acetonitrile, a 3 min gradient from 80.0 to $35.0 \%(\mathrm{v} / \mathrm{v})$ acetonitrile, and a final 3.5 min gradient from 35.0 to $1.5 \%(\mathrm{v} / \mathrm{v})$ acetonitrile.

Glucosinolate identification and quantification

Identification of most of the HPLC peaks was based on a comparison of retention time and UV absorption spectrum as determined on a diode-array detector with those of purified standards. To quantify the amount of gsl we used the standard methods reported by Brown et al. (2003). Briefly, we ran the standard sinigrin (Sigma-Aldrich) for each independent experiment to calibrate the instrument. We produced a standard curve using the sinigrin standard at a series of concentrations and the slope was computed by performing a regression analysis. The quantification of each individual gsl present in the sample was calculated from HPLC peak areas using the sinigrin standard as a reference. Then, the data were corrected using published UV response factors for different types of gsl (ISO-9167, 1992), and the response factors for indolic gsl were originated from Fiebig and Arens (1992). Quantities results are given as

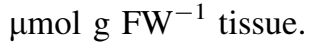

The value shown for each variety is based on the mean of the three extractions for that variety. The list of compound identities based on HPLC is shown in Table 2 . The analysis of variance $F$-test and student's $t$-test were used for sample means comparisons among and between different crops or different gsl (Steel et al. 1997).

\section{HPLC-MS/MS assay}

The identity of three of the gsl (No. 5, No. 6 and No. 8) was further confirmed by a Finnigan LTQ 2D

Table 2 List of glucosinolates found in 82 varieties of Brassica rapa

\begin{tabular}{lllll}
\hline Ret. time & Abbr. & Common name & Chemical name & Type \\
\hline 7 & 4OHB & Progoitrin & 2-Hydroxy-3-butenyl glucosinolate & Aliphatic glucosinolate \\
7.5 & 3PREY & Sinigrin & 2-Propenyl glucosinolate & Aliphatic glucosinolate \\
8.6 & 5OHP & Gluconapoleiferin & 2-Hydroxy-4-pentenyl glucosinolate & Aliphatic glucosinolate \\
8.8 & 5MSO & Glucoalyssin & 5-Methylsulphinylpentyl glucosinolate & Aliphatic glucosinolate \\
9 & 4CMP & & 2-Methyl-2-propenyl glucosinolate & Aliphatic glucosinolate \\
10.5 & 4BTEY & Gluconapin & 3-Butenyl glucosinolate & Aliphatic glucosinolate \\
11.5 & 4OHI3M & 4-Hydroxyglucobrassicin & 4-Hydroxy-3-indolymethyl glucosinolate & Indolic glucosinolate \\
11.7 & 4BTY & Glucocochlearin & $n$-Butyl glucosinolate & Aliphatic glucosinolate \\
12.5 & 5PTEY & Glucobrassicanapin & 4-Pentenyl glucosinolate & Aliphatic glucosinolate \\
13 & 4MTB & Glucoerucin & 4-Methylthiobutyl glucosinolate & Aliphatic glucosinolate \\
13.3 & I3M & Glucobrassicin & 3-Indolymethyl glucosinolate & Indolic glucosinolate \\
13.8 & GNST & Gluconasturtiin & 2-Phenylethyl glucosinolate & Aromatic glucosinolate \\
14.2 & 4MOI3M & 4-Methoxyglucobrassicin & 4-Methoxy-3-indolylmethyl glucosinolate & Indolic glucosinolate \\
14.5 & NMOI3M & Neoglucobrassicin & $N$-methoxy-3-indolymethyl glucosinolate & Indolic glucosinolate \\
\hline
\end{tabular}

The retention time of the peak when it shows in the 20-min HPLC program described in experimental; The abbreviation name of the compound used in supplementary Table 1 
linear Ion Trap Mass Spectrometer system (Thermo Electron Corporation). The LC-MS/MS system consisted of a Finnigan Surveyor LC pump which has a $4 \mu \mathrm{m}$ (Synergi Hydro RP 80, $150 \mathrm{~mm} \times 3 \mathrm{~mm}$ ) column, a Finnigan Surveyor LC autosampler, a Finnigan Surveyor PDA detector, and a Finnigan LTQ mass spectrometer.

HPLC separation was performed at a constant flow rate of $0.4 \mathrm{ml} \mathrm{min}^{-1}$ with a gradient from 0 to $100 \%$ of solvent B in 30 min before column re-equilibration. Solvent A was $13 \mathrm{mM}$ ammonium acetate $(\mathrm{pH}$ 5.5) and solvent B was methanol. A linear-gradient mobile phase from $100 \%(\mathrm{v} / \mathrm{v})$ of solvent $A$ to $15 \%$ $(\mathrm{v} / \mathrm{v})$ of solvent B in $3 \mathrm{~min}$, to $55 \%$ of solvent B (v/v) in $4 \mathrm{~min}$, to $100 \%(\mathrm{v} / \mathrm{v})$ of solvent $\mathrm{B}$ in $13 \mathrm{~min}, 2 \mathrm{~min}$ at $100 \%(\mathrm{v} / \mathrm{v})$ of solvent $\mathrm{B}$, and returned to $100 \%(\mathrm{v} / \mathrm{v})$ of solvent $\mathrm{A}$ in 8 min was used for elution.

Both positive and negative ion tandem mass spectrometry (MS/MS) were conducted to detect gsl with selected reaction monitoring (SRM). The scan event cycle used a full scan mass spectrum with a range of 250.00 to 900.00 mass/charge (m/z) and two corresponding data-dependent MS/MS events. The most intense ions detected during full scan MS triggered data dependent scanning. Data dependent scanning was performed without the use of a parent ion list. The microscan count for full and MS/MS scan events were set to unity and a repeat count for dynamic exclusion was set to three for verification. MS/MS activation parameters used an isolation width of $2.0 \mathrm{~m} / \mathrm{z}$, minimum signal threshold of 500.0 counts, default charge state of 2 , normalized collision energy of 20.0, an activation $Q$ of 0.250 , and an activation time of $30 \mathrm{~ms}$.

\section{Data interpretation}

The data from HPLC-MS/MS were processed using Qual Browser of XCalibur (Thermo Electron, San Jose, CA), which provides accurate mass thresholds to filter data. In addition, chemical formula calculator was used to provide chemical formula and saturation values for product ions of gsl. Comparisons between theoretical and experimental product ion spectra further aided in the identification of the gsl structures. The predictive structure was drawn using ACDLABS 8.0. This determination was done at the metabolomics facility of the UCD Genome Center by Dr. Vladimir Tolstikov.

\section{Results and discussion}

Natural variation of glucosinolate composition

To survey natural variation in the gsl of B. rapa, we identified and quantified 14 different gsl present in the young leaves of the different varieties sampled (Fig. 1; Supplementary Tables 1,2). We found a total of nine aliphatic gsl, four indolic gsl and one aromatic gsl. The predominant aliphatic gsl in most varieties contain four-carbon (4-C) side-chains, except for a rapeseed accession from USSR (Acc \#79), which has predominantly sinigrin (No. 2), a three-carbon (3-C) side chain gsl. The composition of indolic gsl was fairly consistent in most of the varieties except a rapeseed accession from Sweden (Acc \#71), which lacked this type of gsl. 2-phenylethyl gsl, also named gluconaturtiin (No. 12), was the only aromatic gsl found in these varieties. All the varieties except a rapeseed accession from Pakistan (Acc \#77) have this aromatic gsl (Supplementary Table 1).

The identity of three of the compounds, with retention times at $9.8,10.5$ and $11.7 \mathrm{~min}$, was in doubt, so they were further analyzed by HPLC-MS. Turnip 'Kenshin Kabu' (Acc \#51), Turnip 'Kamo Kabu' (Acc \#49) and Turnip 'Royal Crown' \#1 (Acc \#60) were used for this determination because they have high content of these compounds (Supplementary Table 1). All three compounds are 4-C aliphatic gsl and their identities could be ascertained based on retention time, UV spectrum and mass spectrum. The results indicate that they are 2-methyl-2-propenyl gsl $(\mathrm{RT}=9.8), 3$-butenyl gsl $(\mathrm{RT}=10.5)$ and $n$-butyl gsl $(\mathrm{RT}=11.7)$. 2-Methyl-2-propenyl gsl (No. 5) and $n$-butyl gsl (No. 8) are not commonly found in Brassica species (Kiddle et al. 2001). The genetic analyses for these compounds have not yet been performed in any species because of their low amounts and their rarity. In this survey, turnip 'Royal Crown \#1' (Acc \#62) was found to have a high amount of 2-methyl-2-propenyl gsl (No. 5); whereas turnip 'Tokyo Top' (Acc \#65) has a high amount of $n$-butyl gsl (No. 8). These two varieties could be used to determine the genetic basis for the synthesis of these two compounds. The other compound, 3butenyl gsl (No. 6), was commonly found in B. rapa in previous reports (Kang et al. 2006; Bellosta et al. 2007; Padilla et al. 2007). 


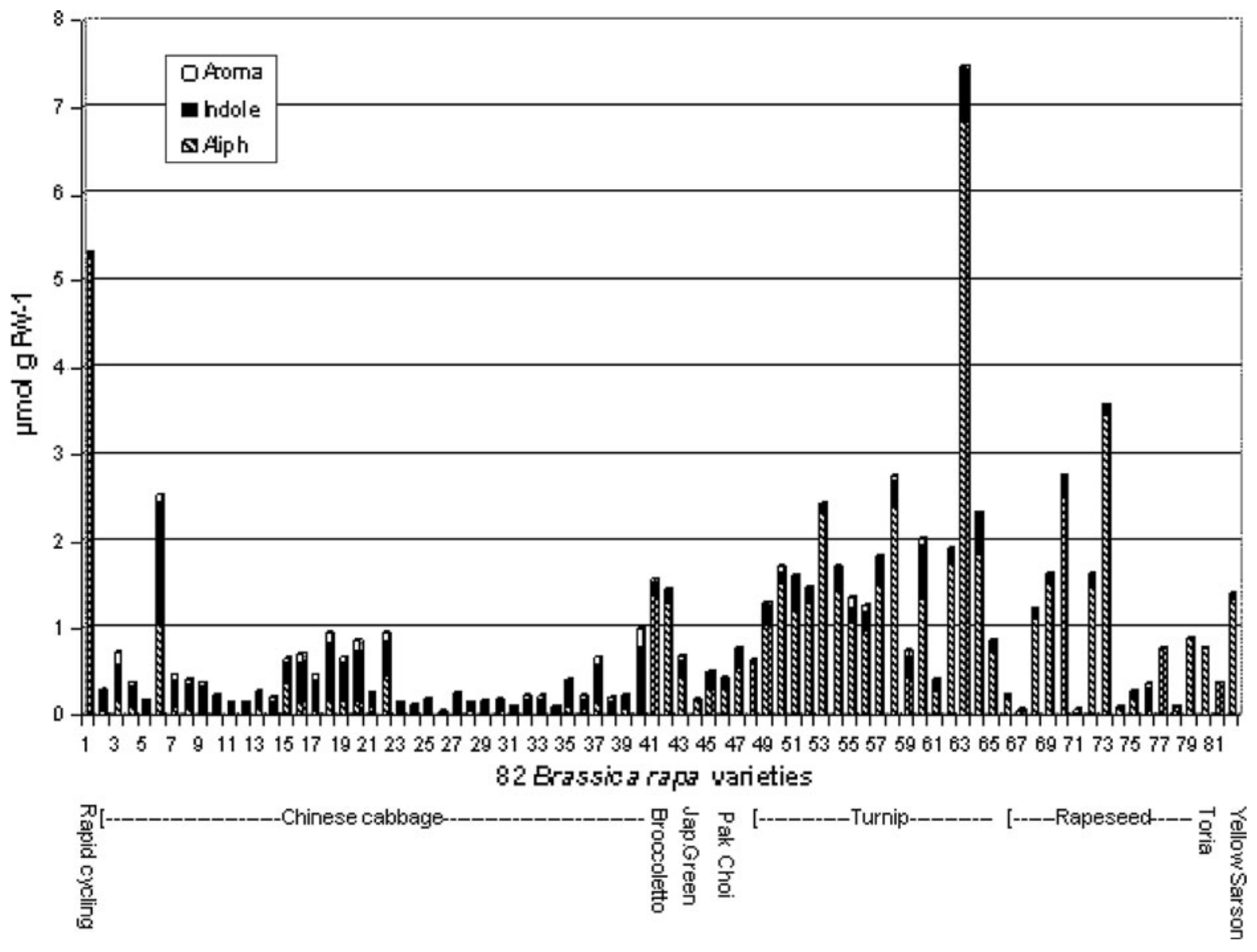

Fig. 1 Relative content of aliphatic, indolic and aromatic gsl accumulation in young leaves of 82 varieties of $B$. rapa. The variety names are listed in Table 1 . The empty region of each bar depicts the average content of total aromatic gsl, the filled

The identities of the main gsl compounds in B. rapa reported in this survey (gluconapin, No. 6; glucobrassicin, No. 11; and gluconasturtiin, No. 12) are consistent with those reported in previous studies (Rosa et al. 1997; Ciska et al. 2000; Skutlarek et al. 2004; Kang et al. 2006; Padilla et al. 2007). Kang et al. (2006) studied in 23 accessions of Chinese cabbage the factors affecting the phenotypic variation of gsl content in two different environments. They detected and quantified only four major gsl: glucobrassicin (No. 11), gluconasturtiin (No. 12), neoglucobrassicin (No. 14) and progoitrin (No. 1). Our results included the quantification of each individual gsl, even those with low concentration for 82 accessions representing most crops in this species. For all these accessions, we found that the major aliphatic gsl region depicts the average content of total indolic gsl the diagonal line region depicts the average content of total aliphatic gsl of each variety

present was gluconapin (No. 6). However, similar to the findings of Kang et al. (2006), progoitrin (No. 1) was in higher amount than gluconapin (No. 6). in the Chinese cabbage accessions we sampled. Padilla et al. (2007) found that gluconapin (No. 6) and glucobrassicanapin (No. 9) were the most abundant aliphatic gsl in turnip greens varieties from northwestern Spain. They found that indolic and aromatic gsl concentrations were low and there were fairly constant in these varieties, which is consistent with our results. In any case, these differences are not surprising considering that age of the plants, organ sampled and growing conditions will affect gsl concentration. Our samples were done at 6 weeks in leaves of plants grown at the greenhouse. It is expected that changes in gsl concentration but not profile will occur in older plants 
and those grown in the field, specially for indolic gsl that are the most affected by environment (Kang et al. 2006).

Natural variation of glucosinolate concentration

Gsl quantity varied extensively among varieties and crops (Fig. 1; Supplementary Table 1). The global mean of total aliphatic gsl was $0.697 \mu \mathrm{mol} \mathrm{g} \mathrm{FW}^{-1}$ leaf tissue and the global mean of total indolic and aromatic gsl were 0.213 and $0.035 \mu \mathrm{mol} \mathrm{g} \mathrm{FW}^{-1}$ leaf tissue, respectively. Turnip 'Tokyo Top' (Acc \#63) had the highest content of aliphatic gsl, $6.823 \mu \mathrm{mol}$ $\mathrm{g} \mathrm{FW}^{-1}$ leaf tissue. In contrast, Chinese cabbage 'Matsushima' (Acc \#29) had only $0.011 \mu \mathrm{mol}$ $\mathrm{g} \mathrm{FW}^{-1}$ and Chinese Cabbage Acc \#31, an unknown variety from Taiwan, lacked aliphatic gsl (Fig. 1). The differences of indolic gsl quantities among accessions are not as dramatic as that observed for of aliphatic gsl. The highest content of indolic gsl was $0.820 \mu \mathrm{mol}$ $\mathrm{g} \mathrm{FW}^{-1}$ leaf tissue from Chinese cabbage 'Wong Bok' (Acc \#40); the lowest one was $0.004 \mu \mathrm{mol} g$ $\mathrm{FW}^{-1}$ leaf tissue from an oilseed Sarson accession from Pakistan (Acc \#81). For aromatic gsl, the highest amount was $0.202 \mu \mathrm{mol} \mathrm{g} \mathrm{FW}^{-1}$ leaf tissue, also from Chinese cabbage 'Wong Bok' (Acc \#40); the lowest one was $0.001 \mu \mathrm{mol} \mathrm{g} \mathrm{FW}^{-1}$ leaf tissue from oilseed sarson (Acc \#81) (Fig. 1).

Aliphatic gsl with 3-C, 4-C and 5-C side-chains were found in the accessions sampled, with variable concentrations for each class (Supplementary Table 1). Only fourteen of the accessions analyzed have 3-C side-chain gsl, with an average content of $0.014 \mu \mathrm{mol} \mathrm{g} \mathrm{FW}^{-1}$ leaf tissue. The crop types of these accessions included Chinese cabbage, Japanese greens, Pak choi, turnip, and rapeseed. Rapeseed accession Acc \#79 had the highest content of 3-C side chain gsl $\left(0.833 \mu \mathrm{mol} \mathrm{g} \mathrm{FW}{ }^{-1}\right.$ leaf tissue) but very low content of 4-C side chain gsl $\left(0.017 \mu \mathrm{mol} \mathrm{\textrm {gW } ^ { - 1 }}\right.$ leaf tissue). The global mean of 4-C side-chain gsl in all accessions was $0.470 \mu \mathrm{mol}$ $\mathrm{g} \mathrm{FW}^{-1}$ leaf tissue, the predominant class of aliphatic gsl in the accessions sampled. The highest amount observed was $5.219 \mu \mathrm{mol} \mathrm{g} \mathrm{FW}^{-1}$ leaf tissue for a doubled haploid 'rapid cycling' line from Wisconsin (Acc \#1) whereas Acc \#31, a Chinese cabbage variety, lacked completely 4-C side chain gsl. The 5-C sidechain gsl quantities are lower than those for the 4-C sidechain gsl in most of the varieties, with the global mean of $0.213 \mu \mathrm{mol} \mathrm{g} \mathrm{FW}^{-1}$ leaf tissue, except for some varieties of Chinese cabbage (Acc\# 40), turnip (Acc $\# 53$ and 54), and rapeseed (Acc \#68 and 79). Acc \#63, turnip 'Tokyo Top', had the highest content of 5-C sidechain gsl, $3.324 \mu \mathrm{mol} \mathrm{g} \mathrm{FW}^{-1}$ leaf tissue, while its 4-C

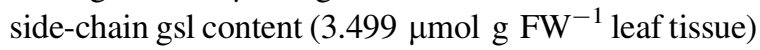
was also relatively high compared to other accessions.

3-Butenyl gsl (gluconapin, No. 6) and 4-pentenyl gsl (glucobrassicanapin, No. 9) were the predominant aliphatic gsl in most of the $B$. rapa varieties. The means of these two compounds are 0.406 and $0.205 \mu \mathrm{mol} \mathrm{g} \mathrm{FW}^{-1}$ leaf tissue, respectively, constituting $\sim 58$ and $29 \%$ of total aliphatic gsl in leaves. $\mathrm{N}$-methoxy-3-indolymethyl gsl (neoglucobrassicin, No. 14) and 3-indolymethyl gsl (glucobrassicin, No. 11) were the predominant indolic gsl in most varieties. The means of these two compounds are 0.120 and $0.085 \mu \mathrm{mol} \mathrm{g} \mathrm{FW}^{-1}$ leaf tissue, respectively, which constitute $\sim 56$ and $40 \%$ of total indolic gsl.

\section{Crop specificity}

The main crops surveyed in this study include Chinese cabbage, broccoleto, Pak choi and other leafy vegetables, turnip, sarson and rapeseed (Table 1; Fig. 1). We did not find crop-specific gsl, however, after testing crops which included more than ten varieties, such as Chinese cabbage, turnip and rapeseed, we found significant differences in the mean for total gsl $(P \ll 0.01)$. Turnip has higher gsl content than Chinese cabbage and rapeseed, but the difference between the last two crops was not significant. The mean total aliphatic gsl in Chinese cabbage, turnip and rapeseed was significantly different $(P \ll 0.01)$. Total aliphatic gsl of Chinese cabbage was significantly lower than that of rapeseed and turnip $(P=0.05)$. However, the total aliphatic gsl of rapeseed and turnip was not significantly different. The methylsufinylalkyl/alkenyl (MSO/ ALK) means were also significantly different in these three crops $(P<0.05)$, but the difference was only significant between that of Chinese cabbage and turnip $(P<0.05)$.

In general, aliphatic gsl content in Chinese cabbage is significantly lower than indolic gsl content $(P<0.01)$. Pak Choi does not have a high amount of aliphatic gsl either, although its aliphatic gsl content is higher than its indolic gsl content (Fig. 1; Supplementary Table 1). Broccoleto, turnip and rapeseed all have significantly higher aliphatic gsl content than indolic 
gsl content $(P \ll 0.01)$, and the main aliphatic gsl for broccoleto and turnip are both 4-C and 5-C side-chain gsl. Some of the rapeseed varieties tend to have high content of 3-C side-chain gsl. Compared to other crops, Chinese cabbage and turnip have significantly higher content of aromatic gsl $(P<0.01)$.

\section{Crop domestication}

Although we did not find crop specific gsl, there were significant differences in total and aliphatic gsl content. These might be related to the domestication of some of these crops considering that gsl derivatives influence their taste and aroma. Chinese cabbage originated in China over 6,000 years ago and was introduced to Korea in the fifteenth century and into Japan in the early twentieth century. It has been suggested that Chinese cabbage may have resulted from hybridization between pak-choi and turnip (Li 1982; Song et al. 1988). The exact place where turnip was domesticated is unknown. It either originated from "primitive types" in central Asia or derived from two independent domestication events in Europe and Asia (McGrath and Quiros 1992). Rapeseed was distributed from northern Europe to China and Korea as early as 2,000 years ago, with the primary center of diversity in the Himalayan region (Sovero 1993). Thus geographically separate domestication of these crops might explain in part the differences observed in their gsl content.

Glucosinolate biosynthetic pathway controlling loci

Our understanding on the genetic control of gsl composition is fairly extensive thanks to the model plant A. thaliana (Halkier and Gershenzon 2006). Most of the loci controlling the biosynthetic pathway for aliphatic gsl in A. thaliana and B. oleracea have been described previously (Kroymann et al. 2001; Kliebenstein et al. 2001a, b; Li and Quiros 2002, 2003; Gao et al. 2004; Textor et al. 2004; Field et al. 2004). Three partially redundant MAM (methylthioalkyl malate synthase) family genes (MAM1, MAM2 and $M A M-L)$ in the $G S$-Elong locus control the variation in side chain length of aliphatic gsl in A. thaliana. Functional analysis demonstrated that $M A M 1$ catalyze the first two elongation cycles for the synthesis of 3-C and 4-C gsl. MAM-L carries out the subsequent elongation cycles forming 5 to 8 -C long chain gsl. $M A M 2$ is assumed to control the 3-C gsl synthesis, but this activity has not been functionally demonstrated (Textor et al. 2004; Field et al. 2004). In B. oleracea, Bogsl-Elong and Bogsl-Pro are suggested to control the elongation cycle ( $\mathrm{Li}$ and Quiros 2002; Gao et al. 2004). B. rapa is a rich source of 5-C aliphatic gsl. This leads to the hypothesis that $B$. rapa also has orthologs for all these three genes in the MAM family.

The effect of three loci on side chain modification of aliphatic gsl in B. rapa could be ascertained for the 82 varieties tested. $\mathrm{Brgsl}$-OX controls the conversion of methylthioalkyl to methylsulfinylalkyl gsl. Most varieties carry out this conversion efficiently and typically contain at least as much methylsulfinylalkyl as methylthioalkyl gsl in the leaves. However, Acc \#4, 13, 37, 40, 46, 58, 64 and 76 all have significantly higher $(P<0.01)$ concentrations of methylthioalkyl than methylsulfinylalkyl gsl (Supplementary Table 2), indicating that they are impaired in this conversion and presumably contain a different Brgsl-OX allele than the other varieties. Brgsl-Alk is responsible for the conversion of methylsufinylalkyl to alkenyl gsl. Most varieties carry out this conversion also very efficiently and typically contain at least twice as much alkenyl as methylsulfinylalkyl gsl in the leaves except for Acc $\# 14$ and $22(P<0.01)$ (Supplementary Table 2). Brgsl-OH controls the production of hydroxylalkenyl gsl (D. Kliebenstein, personal communication). Most varieties carry out this conversion inefficiently and typically contain at most half as much hydroxyalkenyl as alkenyl gsl in the leaves. However, Acc \#2, 14, 22, $28,30,38,55$ and 76 all have significantly higher concentrations of hydroxyalkenyl than alkenyl gsl $(P<0.01)$ (Supplementary Table 2$)$, suggesting that they may contain a more efficient $\mathrm{Brgsl}-\mathrm{OH}$ allele than the other varieties.

Sulforaphane, the isothiocyanate derived from 4-methylsulphinylbutyl gsl (glucoraphanin) commonly found in broccoli, is of great interest because of its role of inducing Phase II enzymes exerting blocking effects on carcinogens (Mithen 2001). However, contrary to broccoli, none of the accessions in the survey have glucoraphanin, which indicates that only functional Brgsl-Alk alleles are present in $B$. rapa. Conventional plant breeding, including the synthesis of artificial B. napus by hybridizing $B$. rapa and $B$. oleracea, could be used to replace functional Brgsl-Alk alleles with their non-functional homologs 
from B. oleracea (broccoli). Alternatively, other approaches to develop a variety of $B$. rapa containing this desirable compound is to produce Brgsl-Alk knockout lines to efficiently accumulate glucoraphanin in the side-chain modification pathway, or use gene silencing methods such as RNAi to accomplish the same objective. The genetic approaches described above could also be applied to manipulate content of other gsl. For instance, disrupting the functional Brgsl-OH allele in B. rapa could abolish the accumulation of the detrimental compound progoitrin (No. 1). The study of natural gsl variation in B. rapa provides valuable information for answering questions about the biosynthesis, evolution and function of these interesting natural products in this species.

\section{Conclusions}

The main contributions of this study are: (1) Determination of gsl profiles and quantification of these compounds in representatives of most crops of in B. rapa. (2) Identification of new compounds not reported before for $B$. rapa. These include 2-methyl-2propenyl (No. 5) and $n$-butyl gsl (No. 8), present in some of the varieties. (3) Although there are no crop specific gsl, there are significant differences in total amounts for these compounds. For example the leafy Chinese cabbages contain higher indolic gsl content than the rest of the B. rapa crops, whereas broccoleto and two oilseed vegetables, turnip and rapeseed, all have much higher aliphatic gsl content than the rest of the crops.

Acknowledgments The research was supported by USDAIFAFS grant \# 00-52100-9683 "Development of Genomic Tools and Resources for Brassica", and by the National Research Initiative of the USDA Cooperative State Research, Education and Extension Service, grant number \#200x-35301-15886 "Cloning and characterization of the major glucosinolate genes involved in the aliphatic glucosinolate pathway of Brassica crops"). We acknowledge the assistance from Mr. Vincent D'Antonio raising the plants, to Dr. Dan Qiu for gsl analysis and to Mr. J. Erron Haggard for proofreading the manuscript. We also thank Dr. Daniel Kliebenstein and Dr. Vladmir Tolstikov for aid in compound identification.

Open Access This article is distributed under the terms of the Creative Commons Attribution Noncommercial License which permits any noncommercial use, distribution, and reproduction in any medium, provided the original author(s) and source are credited.

\section{References}

Barillari J, Iori R, Rollin P, Hennion F (2005) Glucosinolates in the subantarctic crucifer kerguelen cabbage (Pringlea antiscorbutica). J Nat Prod 68:234-236

Bellosta N, Sorensen JC, Sorensen H (2007) Profiling glucosinolates in vegetative and reproductive tissues of four Brassica species of the U-triangle for their biofumigation potential. J Sci Food Agirc 87:1586-1594

Branca F, Li G, Goyal S, Quiros CF (2002) Survey of aliphatic glucosinolates in Sicilian wild and cultivated Brassicaceae. Phytochemistry 59:717-724

Brown PD, Tokuhisa JG, Reichelt M, Gershenzon J (2003) Variation of glucosinolate accumulation among different organs and developmental stages of Arabidopsis thaliana. Phytochemistry 62:471-481

Cipollini DF, Busch JW, Stowe KA, Simms EL, Bergelson J (2003) Genetic variation and relationships of constitutive and hervivore-induced glucosinolates, trypsin inhibitors, and herbivore resistance in Brassica rapa. J Chem Ecol 29:285-302

Ciska E, Martyniak-Przybyszewska B, Kozlowska H (2000) Content of glucosinolates in cruciferous vegetables grown at the same site for two years under different climatic conditions. J Agric Food Chem 48:2862-2867

Fiebig HJ, Arens M (1992) Glucosinolates (HPLC method)—survey by a working party of the Dgf, 128th report-German standard methods for investigation of fats, fatty products, tensides and related materials, 98th report-analysis of fat rawmaterials. 12. Fett Wissenschaft Technologie-Fat Science Technology, vol 94, pp 199-203

Field B, Cardon G, Traka M, Botterman J, Vancanneyt G, Mithen R (2004) Glucosinolate and amino acid biosynthesis in Arabidopsis. Plant Physiol 135:828-839

Font R, Rio-Celestino MD, Cartea E, Haro-Bailon AD (2005) Quantification of glucosinolates in leaves of leaf rape (Brassica napus ssp. pabularia) by near-infrared spectroscopy. Phytochemistry 66:175-185

Gao M, Li G, Yang B, McCombie BWR, Quiros CF (2004) Comparative analysis of a Brassica BAC clone containing several major aliphatic glucosinolate genes with its corresponding Arabidopsis sequence. Genome 47:666-679

Halkier BA, Gershenzon J (2006) Biology and biochemistry of glucosinolates. Ann Rev Plant Biol 57:303-333

ISO 9167-1 (1992) Rapeseeds-determination of glucosinolates content. Part 1. Method using high performance liquid chromatography. International Organization for Standardization. Geneva, Switzerland

Kang JY, Ibrahim KE, Kim DH, Kang WJ, Juvik JA (2006) Genetic and environmental variation of glucosinolate content in Chinese cabbage. HortScience 41:1382-1385

Kiddle G, Bennett RN, Botting NP, Davidson NE, Robertson AAB, Wallsgrove RM (2001) High-performance liquid chromatographic separation of natural and synthetic desulphoglucosinolates and their chemical validation by UV, NMR and chemical ionisation-MS methods. Phytochem Anal 12:226-242

Kliebenstein DJ, Kroymann J, Brown P, Figuth A, Pedersen D, Gershenzon J, Mitchell-Olds T (2001a) Genetic control of 
natural variation in Arabidopsis glucosinolate accumulation. Plant Physiol 126:811-825

Kliebenstein DJ, Lambrix VM, Reichelt M, Gershenzon J, Mitchell-Olds T (2001b) Gene duplication in the diversification of secondary metabolism: tandem 2-oxoglutaratedependent dioxygenases control glucosinolate biosynthesis in Arabidopsis. Plant Cell 13:681-693

Koroleva OA, Davies A, Deeken R, Thorpe MR, Tomos AD, Hedrich R (2000) Identification of a new glucosinolaterich cell type in Arabidopsis flower stalk. Plant Physiol 124:599-608

Kroymann J, Textor S, Tokuhisa JG, Falk KL, Bartram S, Gershenzon J, Mitchell-Olds T (2001) A gene controlling variation in Arabidopsis glucosinolate composition is part of the methionine chain elongation pathway. Plant Physiol 127:1077-1088

Li CW (1982) The origin, evolution, taxonomy and hybridization of Chinese cabbage. In: Talekar NS, Griggs TD (eds) Chinese cabbage, Proceedings of first international 1 AVRDC symposium 1981, Taiwan, pp 1-10

Li G, Quiros CF (2002) Genetic analysis, expression and molecular characterization of Bogsl-ELONG, a major gene involved in the aliphatic glucosinolate pathway of Brassica species. Genetics 162:1937-1943

Li G, Quiros CF (2003) In planta side-chain glucosinolate modification in Arabidopsis by introduction of dioxygenase Brassica homolog Bogsl-ALK. Theor Appl Genet 106:1116-1121

McGrath JM, Quiros CF (1992) Genetic diversity at isozyme and RFLP loci in Brassica campestris as related to crop type and geographical origin. Theor Appl Genet 83:783-790

Mithen R (2001) Glucosinolates-biochemistry, genetics and biological activity. Plant Growth Reg 34:91-103

Padilla G, Cartea ME, Velasco P, Haro AD, Ordas A (2007) Variation of glucosinolates in vegetable crops of Brassica rapa. Phytochemistry 68:536-545

Petersen BL, Chen S, Hansen CH, Olsen CE, Halkier BA (2002) Composition and content of glucosinolates in developing Arabidopsis thaliana. Planta 214:562-571
Rosa EAS, Heancy RK, Fenwick GR, Portas CAM (1997) Glucosinolates in crop plants. Hort Rev 19:99-215

Siemens DH, Garner SH, Mitchell-Olds T, Callaway RM (2002) Cost of defense in the context of plant competition: Brassica rapa may grow and defend. Ecology 83:505-517

Skutlarek D, Farber H, Lippert F, Ulbrich A, Wawrzum A, Buning-Pfaue H (2004) Determination of glucosinolate profiles in Chinese vegetables by precursor ion scan and multiple reaction monitoring scan mode (LC-MS/MS). Eur Food Res Technol 219:643-649

Song KM, Osborn TC, Williams PH (1988) Brassica taxonomy based on nuclear restriction fragment length polymorphisms (RFLPs).2. Preliminary analysis of subspecies within B. rapa (syn. campestris) and B. oleracea. Theor Appl Genet 76:593-600

Sovero M (1993) Rapeseed, a new oilseed crop for the United States. In: Janick J, Simon JE (eds) New crops. Wiley, New York, pp 302-307

Steel RGD, Torrie JH, Dickey DA (1997) Principles and procedures of statistics, a biometrical approach, 3rd edn. McGrawHill series in probability and statistics. McGraw-Hill, Boston

Textor S, Bartram S, Kroymann J, Falk KL, Hick A, Pickett JA, Gershenzon J (2004) Biosynthesis of methionine-derived glucosinolates in Arabidopsis thaliana: recombinant expression and characterization of methylthioalkylmalate synthase, the condensing enzyme of the chain-elongation cycle. Planta 218:1026-1035

Tian Q, Rosselot RA, Schwartz SJ (2005) Quantitative determination of intact glucosinolates in broccoli, broccoli sprouts, Brussels sprouts, and cauliflower by highperformance liquid chromatography-electrospray ionization-tandem mass spectrometry. Anal Biochem 343:93-99

Windsor AJ, Reichelt M, Figuth A, Svatos A, Kroymann J, Kleibenstein DJ, Gershenzon J, Mitchell-Olds T (2005) Geographic and evolutionary diversification of glucosinolates among near relatives of Arabidopsis thaliana (Brassicaceae). Phytochemistry 66:1321-1333 